\section{Physical activity in Brazil: a systematic review}

\author{
Atividade física no Brasil: uma revisão sistemática
}

\begin{abstract}
The purpose of this study, based on a systematic literature review, was to describe the prevalence of physical activity (or inactivity) in the Brazilian population. The databases consulted were: LILACS, SciELO, MEDLINE, Web of Science, and the Google Scholar portal. The terms "physical activity", "physical exercise", "physical inactivity", "sedentary" "Brazil", and "Brazilian" were used in the search. Overall, 47 studies (all cross-sectional) with random samples were found, and in 26 studies physical activity was the main variable. Only two studies were published before the year 2000, as compared to 12 in 2008 alone. The studies were heavily concentrated in the South and Southeast of Brazil, and there were few studies on physical activity in children and adolescents. In all the studies, physical activity was measured subjectively, mainly with questionnaires, and the most widely studied domain was leisure time. The criteria for defining physical activity varied widely, as did prevalence. The study highlighted the need for standardization of instruments, criteria, and nomenclature in epidemiological studies on physical activity.
\end{abstract}

Motor Activity; Exercise; Review
Samuel C. Dumith ${ }^{1}$

\section{Introduction}

Research in the field of physical activity has grown significantly in recent years. A search in MEDLINE/PubMed for articles containing the term "physical activity" in the title and/or abstract showed that the number of articles published since 2000 (nearly 30 thousand) has exceeded the total published in the entire 20th century (approximately 20 thousand). Considering all articles launched in the respective databases (independently of subject), for every 200 articles published, one contains the term "physical activity" in the title and/or abstract (i.e., 5.3 per thousand). In Brazil, a recent review of the evolution in epidemiological research in physical activity also shows this same trend 1 .

Although the health benefits of physical activity are well established in the literature ${ }^{2}$, the World Health Organization (WHO) 3 estimates that some $17 \%$ of the world population practices no physical activity whatsoever, and approximately $60 \%$ fail to reach the minimum criterion (2.5 hours or 150 minutes per week) to be considered physically active. The most comprehensive study in the world on physical activity was conducted in 2002-2003, with 212,021 adults (18-69 years of age) from 51 countries (mostly low and medium income), including Brazil. The overall prevalence of physical inactivity (non-weighted mean for all the countries) was $18 \%$, as compared 
to $25 \%$ and $30 \%$, respectively for Brazilian men and women 4 .

There have been various studies on physical activity in Brazil, but mostly in specific populations, i.e., in a given location. In 2003, the National Cancer Institute (INCA), coordinated by the Ministry of Health, conducted the first national survey (which however included only 15 State capitals) containing information on physical activity in the population 15 to 69 years of age 5 . In this survey, overall prevalence of insufficient physical activity was $37 \%$, varying from $28 \%$ in Belém (Pará State) to 55\% in João Pessoa (Paraíba State).

In 2005, the Brazilian Ministry of Health launched the program Surveillance of Risk Factors for Chronic Non-Communicable Diseases by Telephone Survey (VIGITEL). This nationwide survey has been conducted annually since 2006 in all the State capitals plus the Federal District. According to the most recent available data (for 2008), physical inactivity (defined as no leisuretime physical activity in the previous three months, absence of intense effort at work, inactive commuting to work, and no involvement in heavy housecleaning) reaches $26.3 \%$ of the Brazilian population $(29.5 \%$ of men and $23.5 \%$ of women) 6 . Additionally, only $16.4 \%$ (20.6\% of men and $12.8 \%$ of women) met the criteria to be considered active during their leisure time (30 minutes of moderate activity at least 5 times a week or 20 minutes of vigorous activity at least 3 times a week). Comparing the surveys from 20067 and 2007 , the prevalence of physical inactivity decreased slightly (a drop of 2.9 percentage points), while sufficient leisure-time activity increased slightly (by 1.5 percentage points), with this increase occurring mainly in males.

The main objective of the current study, based on a systematic literature review, was to describe the prevalence of physical activity (or inactivity) in Brazil. The article describes the studies based on the following characteristics: year of publication, place of data collection, origin and size of sample, age bracket, research instrument, target domain and behavior, and criteria used to define physical activity/inactivity.

\section{Methodology}

In order to achieve the study's objectives, a search was performed in the following electronic databases: LILACS (http://www.bireme.br), SciELO (http://www.scielo.org), MEDLINE (http://www. pubmed.com), and Web of Science (http://www. isiknowledge.com). The Google Scholar search portal (http:/ / www.scholar.google.com) was also accessed to detect other publications not indexed in the above-mentioned databases. The terms used to search scientific articles were (in Portuguese): "physical activity" or "physical exercise" or "physical inactivity" or "sedentary" combined with "Brazil" or "Brazilian". The terms were also searched in English. No limitations were set as to date, language, or sample size.

To be included in the present review, the studies needed to have some information on the prevalence of physical activity and/or physical inactivity in the Brazilian population, and the sample had to be representative of the target population, i.e., with random sampling. With the references retrieved from each database, a reading of the titles was performed. Among references selected as possibly eligible, the abstracts were read. Based on the selected abstracts, a search was conducted for each article's full text to assess whether it met the inclusion criteria. Among the selected articles, the references were examined to identify any other publication that met the study's entrance requirements. When there was more than one article with data from the same study, the one pertaining to the original publication was selected.

The reference searches were closed on January 1, 2009. Thus, articles that were published and/or indexed in the respective databases after this date were not included in this study. For the data analysis, the studies are described according to each target factor's absolute frequency.

\section{Results}

Forty-seven articles were found that met the inclusion criteria (see the Methodology section). In order to facilitate the description, articles were divided into two tables: physical activity was studied as the main variable (26 studies; Table 1); physical activity was studied jointly with other factors, or as a secondary variable (21 studies; Table 2). All the articles used a cross-sectional design, so the tables did not include this information.

The first Brazilian study that approached prevalence of physical activity was published in 1990 9, and the second in 1993 10. However, the first study with physical activity as the main variable was not published until 200011 . Recent years have witnessed a clear growth in publications, as shown in Figure 1. In the year 2008 alone, the number of articles nearly doubled that of 2007, with an average of one article published per month.

As for place of data collection, one study included all the Brazilian State capitals 8 and two 
Table 1

Studies in which prevalence of physical activity and/or inactivity was assessed as the principal variable ( $n=26$ studies), Brazil.

\begin{tabular}{|c|c|c|c|c|c|c|c|c|c|}
\hline $\begin{array}{l}\text { Author/ } \\
\text { Year }\end{array}$ & City/State & Sample type & $\mathbf{N}$ & $\begin{array}{l}\text { Age } \\
\text { bracket } \\
\text { (years) }\end{array}$ & $\begin{array}{l}\text { Instru- } \\
\text { ment }\end{array}$ & $\begin{array}{l}\text { Domain of } \\
\text { physical } \\
\text { activity }\end{array}$ & $\begin{array}{l}\text { Study } \\
\text { variable }\end{array}$ & Definition & Prevalence \\
\hline $\begin{array}{l}\text { Mello et al. } \\
11 / 2000\end{array}$ & $\begin{array}{l}\text { São Paulo/ } \\
\text { SP }\end{array}$ & Population & 1,000 & $\geq 20$ & $\begin{array}{l}\text { Own } \\
\text { question- } \\
\text { naire }\end{array}$ & Not mentioned & $\begin{array}{l}\text { Regular } \\
\text { physical } \\
\text { activity }\end{array}$ & Not mentioned & Overall: $31.3 \%$ \\
\hline $\begin{array}{l}\text { Gomes et al. } \\
52 / 2001\end{array}$ & $\begin{array}{c}\text { Rio de } \\
\text { Janeiro/RJ }\end{array}$ & Population & 4,331 & $\geq 12$ & $\begin{array}{l}\text { Own } \\
\text { question- a } \\
\text { naire }\end{array}$ & $\begin{array}{l}\text { Leisure, work, } \\
\text { and commuting }\end{array}$ & $\begin{array}{l}\text { Regular } \\
\text { physical } \\
\text { activity }\end{array}$ & $\begin{array}{l}\text { Physical activity } \\
\text { sometimes or always in } \\
\text { previous month }\end{array}$ & $\begin{array}{l}\text { Overall (men: } 40.2 \% \text {; } \\
\text { women: } 22.2 \% \text { ) } \\
\text { Leisure-time (men: } 36.7 \% \text {; } \\
\text { women: } 19.2 \% \text { ) }\end{array}$ \\
\hline \multirow[t]{3}{*}{$\begin{array}{l}\text { Matsudo et } \\
\text { al. } 33 / 2002\end{array}$} & \multirow[t]{3}{*}{$\begin{array}{l}\text { São Paulo/ } \\
\text { SP }\end{array}$} & \multirow[t]{3}{*}{ Population } & \multirow[t]{3}{*}{2,001} & \multirow[t]{3}{*}{$14-77$} & \multirow[t]{3}{*}{$\begin{array}{l}\text { IPAQ - short } \\
\text { version }\end{array}$} & \multirow{3}{*}{$\begin{array}{l}\text { Leisure, work, } \\
\text { household and } \\
\text { commuting }\end{array}$} & $\begin{array}{l}\text { Sedentary } \\
\text { lifestyle }\end{array}$ & $\begin{array}{c}\text { No weekly physical } \\
\text { activity }\end{array}$ & $\begin{array}{c}\text { Overall: } 8.8 \% \text { (men: 9.7\%; } \\
\text { women: } 8.0 \% \text { ) }\end{array}$ \\
\hline & & & & & & & $\begin{array}{l}\text { Irregular } \\
\text { physical } \\
\text { activity }\end{array}$ & $\begin{array}{c}\text { Does not meet criteria } \\
\text { for regular physical } \\
\text { activity }\end{array}$ & $\begin{array}{c}\text { Overall: } 37.6 \% \text { (men: } \\
35.9 \% \text {; women: } 39.3 \% \text { ) }\end{array}$ \\
\hline & & & & & & & $\begin{array}{l}\text { Regular } \\
\text { physical } \\
\text { activity }\end{array}$ & $\begin{array}{c}\geq 150 \text { minutes/week } \\
\text { of moderate physical } \\
\text { activity or } \geq 60 \text { minutes/ } \\
\text { week of vigorous } \\
\text { physical activity }\end{array}$ & $\begin{array}{l}\text { Overall: 53.5\% (men: } \\
\text { 44.5\%; women: } 52.7 \% \text { ) }\end{array}$ \\
\hline $\begin{array}{l}\text { Hallal et al. } \\
34 / 2003\end{array}$ & Pelotas/RS & Population & 3,182 & $\geq 20$ & $\begin{array}{l}\text { IPAQ - short } \\
\text { version }\end{array}$ & $\begin{array}{l}\text { Leisure, work, } \\
\text { household and } \\
\text { commuting }\end{array}$ & $\begin{array}{l}\text { Physical } \\
\text { inactivity }\end{array}$ & $\begin{array}{c}<150 \text { minutes/week } \\
\text { of moderate physical } \\
\text { activity or }<60 \text { minutes/ } \\
\text { week of vigorous } \\
\text { physical activity }\end{array}$ & $\begin{array}{l}\text { Overall: } 41.1 \% \text { (men: } \\
\text { 40.2\%; women: } 41.8 \% \text { ) }\end{array}$ \\
\hline \multirow[t]{2}{*}{$\begin{array}{l}\text { Monteiro et } \\
\text { al. } 12 / 2003\end{array}$} & \multirow{2}{*}{$\begin{array}{l}\text { Southeast } \\
\text { and } \\
\text { Northeast of } \\
\text { Brazil }\end{array}$} & \multirow[t]{2}{*}{ Population } & \multirow[t]{2}{*}{11,033} & \multirow[t]{2}{*}{$\geq 20$} & \multirow{2}{*}{$\begin{array}{l}\text { Own } \\
\text { question- } \\
\text { naire }\end{array}$} & \multirow[t]{2}{*}{ Leisure } & \multirow[t]{2}{*}{$\begin{array}{l}\text { Physical } \\
\text { activity }\end{array}$} & $\begin{array}{l}30 \text { minutes at least } \\
1 \text { day/week }\end{array}$ & $\begin{array}{l}\text { Overall: } 13.0 \% \text { (men: } \\
\text { 18.2\%; women: } 8.2 \% \text { ) }\end{array}$ \\
\hline & & & & & & & & $\begin{array}{l}30 \text { minutes at least } \\
5 \text { days/week }\end{array}$ & $\begin{array}{c}\text { Overall: } 3.3 \% \text { (men: } 3.5 \% \text {; } \\
\text { women: } 3.2 \% \text { ) }\end{array}$ \\
\hline $\begin{array}{l}\text { Salles-Costa } \\
\text { et al. } 26 / 2003\end{array}$ & $\begin{array}{c}\text { Rio de } \\
\text { Janeiro/RJ }\end{array}$ & $\begin{array}{l}\text { Workers } \\
\text { (university } \\
\text { employees) }\end{array}$ & 3,740 & $20-60$ & $\begin{array}{l}\text { Own } \\
\text { question- } \\
\text { naire }\end{array}$ & Leisure & $\begin{array}{l}\text { Physical } \\
\text { activity }\end{array}$ & $\begin{array}{l}\text { Any physical activity in } \\
\text { the previous } 2 \text { weeks }\end{array}$ & $\begin{array}{c}\text { Overall: } 45.9 \% \text { (men: } \\
\text { 52.2\%; women: } 40.8 \% \text { ) }\end{array}$ \\
\hline $\begin{array}{l}\text { Santos \& } \\
\text { Coelho } \\
27 / 2003\end{array}$ & Joinvile/SC & $\begin{array}{l}\text { Workers } \\
\text { (company } \\
\text { employees) }\end{array}$ & 2,143 & $17-65$ & $\begin{array}{l}\text { Question- } \\
\text { naire } \\
\text { developed } \\
\text { by Pate } \\
\text { (1995) }\end{array}$ & $\begin{array}{l}\text { Leisure, work, } \\
\text { and commuting }\end{array}$ & $\begin{array}{l}\text { Insufficient } \\
\text { physical } \\
\text { activity }\end{array}$ & Not mentioned & $\begin{array}{l}\text { Overall: } 47.1 \% \text { (men: } \\
\text { 33.0\%; women: 62.0\%) }\end{array}$ \\
\hline $\begin{array}{l}\text { Hallal et al. } \\
35 / 2004\end{array}$ & Pelotas/RS & Population & 3,182 & $\geq 20$ & $\begin{array}{l}\text { IPAQ - short } \\
\text { version }\end{array}$ & $\begin{array}{l}\text { Leisure, work, } \\
\text { household and } \\
\text { commuting }\end{array}$ & $\begin{array}{l}\text { Vigorous } \\
\text { physical } \\
\text { activity }\end{array}$ & $\begin{array}{c}\geq 60 \text { minutes/week } \\
\text { of vigorous physical } \\
\text { activity }\end{array}$ & Overall: $29.0 \%$ \\
\hline $\begin{array}{l}\text { Oehlschla- } \\
\text { eger et al. } \\
53 / 2004\end{array}$ & Pelotas/RS & Population & 962 & $15-18$ & $\begin{array}{l}\text { Own } \\
\text { question- } \\
\text { naire }\end{array}$ & $\begin{array}{l}\text { Leisure and } \\
\text { school }\end{array}$ & $\begin{array}{l}\text { Sedentary } \\
\text { lifestyle }\end{array}$ & $\begin{array}{l}\text { Duration }<20 \text { minutes/ } \\
\text { day and frequency } \\
<3 \text { times/week }\end{array}$ & $\begin{array}{l}\text { Overall: } 39.0 \% \text { (boys: } \\
\text { 22.2\%; girls: } 24.5 \% \text { ) }\end{array}$ \\
\hline $\begin{array}{l}\text { Dias-da- } \\
\text { Costa et al. } \\
54 / 2005\end{array}$ & Pelotas/RS & Population & 2,177 & $20-69$ & $\begin{array}{l}\text { Own } \\
\text { question- } \\
\text { naire }\end{array}$ & Leisure & $\begin{array}{l}\text { Physical } \\
\text { inactivity }\end{array}$ & $\begin{array}{c}\text { Energy expenditure in } \\
\text { leisure-time physical } \\
\text { activity }<1,000 \mathrm{kcal} / \\
\text { week }\end{array}$ & $\begin{array}{l}\text { Overall: 80.6\% (men: } \\
\text { 69.1\%; women: 89.4\%) }\end{array}$ \\
\hline \multirow[t]{2}{*}{$\begin{array}{l}\text { Masson et al. } \\
14 / 2005\end{array}$} & \multirow[t]{2}{*}{$\begin{array}{l}\text { São } \\
\text { Leopoldo/ } \\
\text { RS }\end{array}$} & \multirow[t]{2}{*}{$\begin{array}{l}\text { Population (only } \\
\text { women) }\end{array}$} & \multirow[t]{2}{*}{1,026} & \multirow[t]{2}{*}{$20-60$} & \multirow{2}{*}{$\begin{array}{c}\text { Own } \\
\text { question- } \\
\text { naire } \\
\text { (adapted } \\
\text { from Agita } \\
\text { Brasil) }\end{array}$} & \multirow[t]{2}{*}{ Leisure } & $\begin{array}{l}\text { Sedentary } \\
\text { lifestyle }\end{array}$ & $\begin{array}{c}\text { No moderate or } \\
\text { vigorous physical } \\
\text { activity }\end{array}$ & Women: $37.0 \%$ \\
\hline & & & & & & & $\begin{array}{l}\text { Sufficient } \\
\text { physical } \\
\text { activity }\end{array}$ & $\begin{array}{c}\text { Moderate or vigorous } \\
\text { physical activity }>3 \\
\text { times/week }\end{array}$ & Women: $3.6 \%$ \\
\hline $\begin{array}{l}\text { Pitanga } \\
\& \text { Lessa } \\
55 / 2005\end{array}$ & Salvador/BA & Population & 2,292 & $20-90$ & $\begin{array}{l}\text { Own } \\
\text { question- } \\
\text { naire }\end{array}$ & Leisure & $\begin{array}{l}\text { Sedentary I } \\
\text { lifestyle }\end{array}$ & $\begin{array}{l}\text { No leisure-time physical } \\
\text { activity in normal week }\end{array}$ & $\begin{array}{l}\text { Overall: } 72.5 \% \text { (men: } \\
60.4 \% \text {; women: } 82.7 \% \text { ) }\end{array}$ \\
\hline \multirow[t]{2}{*}{$\begin{array}{l}\text { Hallal et al. } \\
42 / 2006\end{array}$} & \multirow[t]{2}{*}{ Pelotas/RS } & \multirow[t]{2}{*}{$\begin{array}{l}\text { Population (1993 } \\
\text { birth cohort) }\end{array}$} & \multirow[t]{2}{*}{4,452} & \multirow[t]{2}{*}{$10-12$} & \multirow{2}{*}{$\begin{array}{l}\text { Own } \\
\text { question- } \\
\text { naire }\end{array}$} & \multirow[t]{2}{*}{$\begin{array}{l}\text { Leisure and } \\
\text { commuting }\end{array}$} & $\begin{array}{l}\text { Sedentary } \\
\text { lifestyle }\end{array}$ & $<300$ minutes/week & $\begin{array}{l}\text { Overall: } 58.2 \% \text { (boys: } \\
49.0 \% \text {; girls: } 67.0 \% \text { ) }\end{array}$ \\
\hline & & & & & & & $\begin{array}{l}\text { Physical } \\
\text { activity }\end{array}$ & $\begin{array}{l}\text { Some leisure-time } \\
\text { physical activity }\end{array}$ & $\begin{array}{l}\text { Overall: } 85.4 \% \text { (boys: } \\
72.9 \% \text {; girls: } 79.0 \% \text { ) }\end{array}$ \\
\hline
\end{tabular}

(continues) 
Table 1 (continued)

\begin{tabular}{|c|c|c|c|c|c|c|c|c|c|}
\hline $\begin{array}{l}\text { Author/ } \\
\text { Year }\end{array}$ & City/State & Sample type & $\mathbf{N}$ & $\begin{array}{l}\text { Age } \\
\text { bracket } \\
\text { (years) }\end{array}$ & $\begin{array}{l}\text { Instru- } \\
\text { ment }\end{array}$ & $\begin{array}{l}\text { Domain of } \\
\text { physical } \\
\text { activity }\end{array}$ & $\begin{array}{c}\text { Study } \\
\text { variable }\end{array}$ & Definition & Prevalence \\
\hline $\begin{array}{l}\text { Arruda } \\
\& \text { Lopes } \\
16 / 2007\end{array}$ & Lages/SC & Schoolchildren & 1,204 & $10-17$ & $\begin{array}{l}\text { Daily } \\
\text { physical } \\
\text { activity log } \\
\text { (adapted } \\
\text { from } \\
\text { Bouchard } \\
\text { et al., 1983) }\end{array}$ & All & $\begin{array}{l}\text { Normal } \\
\text { physical } \\
\text { activity }\end{array}$ & $\begin{array}{l}\text { Energy expenditure } \\
\geq 37.0 \mathrm{kcal} / \mathrm{kg} / \text { day }\end{array}$ & Overall: $70.6 \%$ \\
\hline \multirow[t]{2}{*}{$\begin{array}{l}\text { Azevedo et } \\
\text { al. } 36 / 2007\end{array}$} & \multirow[t]{2}{*}{ Pelotas/RS } & \multirow[t]{2}{*}{ Population } & \multirow[t]{2}{*}{3,100} & \multirow[t]{2}{*}{$\geq 20$} & \multirow[t]{2}{*}{$\begin{array}{l}\text { IPAQ - long } \\
\text { version }\end{array}$} & \multirow[t]{2}{*}{ Leisure } & \multirow[t]{2}{*}{$\begin{array}{l}\text { Physical } \\
\text { activity }\end{array}$} & $\begin{array}{l}\geq 150 \text { minutes/week of } \\
\text { moderate to vigorous } \\
\text { physical activity }\end{array}$ & $\begin{array}{l}\text { Overall: } 27.4 \% \text { (men: } \\
33.9 \% \text {; women: } 22.3 \% \text { ) }\end{array}$ \\
\hline & & & & & & & & $\begin{array}{c}\text { The above criterion } \geq 5 \\
\text { days/week of moderate } \\
\text { physical activity or } 3 \\
\text { days/week of vigorous } \\
\text { physical activity }\end{array}$ & $\begin{array}{l}\text { Overall: } 18.2 \% \text { (men: } \\
22.2 \% \text {; women: } 15.2 \% \text { ) }\end{array}$ \\
\hline $\begin{array}{l}\text { Baretta et al. } \\
37 / 2007\end{array}$ & Joaçaba/SC & Population & 575 & $20-59$ & $\begin{array}{l}\text { IPAQ - short } \\
\text { version }\end{array}$ & $\begin{array}{l}\text { Leisure, work, } \\
\text { household and } \\
\text { commuting }\end{array}$ & $\begin{array}{l}\text { Physical } \\
\text { inactivity }\end{array}$ & Not mentioned & $\begin{array}{l}\text { Overall: } 57.4 \% \text { (men: } \\
55.8 \% \text {; women: } 58.7 \% \text { ) }\end{array}$ \\
\hline $\begin{array}{l}\text { Domingues } \\
\& \text { Barros } \\
15 / 2007\end{array}$ & Pelotas/RS & $\begin{array}{c}\text { Population } \\
\text { (women that gave } \\
\text { birth in 2004) }\end{array}$ & 4,470 & $12-46$ & $\begin{array}{l}\text { Own } \\
\text { question- } \\
\text { naire }\end{array}$ & Leisure & $\begin{array}{l}\text { Physical } \\
\text { activity }\end{array}$ & Not mentioned & $\begin{array}{l}\text { Before pregnancy: } 14.8 \% \text {; } \\
\text { during pregnancy: } 12.9 \%\end{array}$ \\
\hline $\begin{array}{l}\text { Dumith et al. } \\
56 / 2007\end{array}$ & Pelotas/RS & Population & 3,136 & $\geq 20$ & $\begin{array}{l}\text { Own } \\
\text { question- } \\
\text { naire }\end{array}$ & Leisure & $\begin{array}{l}\text { Regular } \\
\text { physical } \\
\text { activity }\end{array}$ & $\begin{array}{l}\geq 20 \text { minutes/day of } \\
\text { moderate to vigorous } \\
\text { physical activity } \\
\geq 3 \text { days/week }\end{array}$ & $\begin{array}{l}\text { Overall: } 29.2 \% \text { (men: } \\
33.6 \% \text {; women: } 25.8 \% \text { ) }\end{array}$ \\
\hline $\begin{array}{l}\text { Gonçalves et } \\
\text { al. } 43 / 2007\end{array}$ & Pelotas/RS & $\begin{array}{l}\text { Population (birth } \\
\text { cohort) }\end{array}$ & 4,452 & $10-12$ & $\begin{array}{l}\text { Own } \\
\text { question- } \\
\text { naire }\end{array}$ & Leisure & $\begin{array}{l}\text { Sedentary } \\
\text { lifestyle }\end{array}$ & $<300$ minutes/week & $\begin{array}{l}\text { Overall: } 68.5 \% \text { (boys: } \\
59.0 \% \text {; girls: } 77.7 \% \text { ) }\end{array}$ \\
\hline $\begin{array}{l}\text { Zaitune et al. } \\
31 / 2007\end{array}$ & $\begin{array}{l}\text { Campinas/ } \\
\text { SP }\end{array}$ & Population & 426 & $\geq 60$ & $\begin{array}{l}\text { Own } \\
\text { question- } \\
\text { naire }\end{array}$ & Leisure & $\begin{array}{l}\text { Sedentary } \\
\text { lifestyle }\end{array}$ & $\begin{array}{l}\text { No weekly physical } \\
\text { activity }\end{array}$ & $\begin{array}{l}\text { Overall: } 70.9 \% \text { (men: } \\
65.8 \% \text {; women: } 74.7 \% \text { ) }\end{array}$ \\
\hline $\begin{array}{l}\text { Bastos et al. } \\
44 / 2008\end{array}$ & Pelotas/RS & Population & 857 & $10-19$ & $\begin{array}{l}\text { Own } \\
\text { question- } \\
\text { naire }\end{array}$ & $\begin{array}{l}\text { Leisure and | } \\
\text { commuting }\end{array}$ & $\begin{array}{l}\text { Insufficient } \\
\text { physical } \\
\text { activity }\end{array}$ & $<300$ minutes/week & $\begin{array}{l}\text { Overall: } 69.8 \% \text { (boys: } \\
52.2 \% \text {; girls: } 81.6 \% \text { ) }\end{array}$ \\
\hline $\begin{array}{l}\text { Benedetti et } \\
\text { al. } 32 / 2008\end{array}$ & $\begin{array}{l}\text { Florianó- } \\
\text { polis/SC }\end{array}$ & Population & 875 & $\geq 60$ & $\begin{array}{l}\text { IPAQ - long } \\
\text { version }\end{array}$ & $\begin{array}{l}\text { Leisure, work, } \\
\text { household and } \\
\text { commuting }\end{array}$ & $\begin{array}{l}\text { Sedentary } \\
\text { lifestyle }\end{array}$ & $\begin{array}{c}<150 \text { minutes/week of } \\
\text { moderate to vigorous } \\
\text { physical activity }\end{array}$ & $\begin{array}{l}\text { Overall: } 40.7 \% \text { (men: } \\
\text { 36.4\%; women: } 45.0 \% \text { ) }\end{array}$ \\
\hline $\begin{array}{l}\text { Cunha et al. } \\
38 / 2008\end{array}$ & Goiânia/GO & Population & 2,002 & $\ddagger 18$ & $\begin{array}{l}\text { Own } \\
\text { question- } \\
\text { naire } \\
\text { (telephone } \\
\text { interviews) }\end{array}$ & $\begin{array}{l}\text { Leisure, work, } \\
\text { household and } \\
\text { commuting }\end{array}$ & $\begin{array}{l}\text { Sedentary I } \\
\text { lifestyle }\end{array}$ & $\begin{array}{c}\text { No leisure-time physical } \\
\text { activity, light effort } \\
\text { at work, no heavy } \\
\text { domestic physical } \\
\text { activity, and active } \\
\text { commuting }<10 \\
\text { minutes/day }\end{array}$ & $\begin{array}{l}\text { Overall: } 50.3 \% \text { (men: } \\
\text { 42.0\%; women: } 55.5 \% \text { ) }\end{array}$ \\
\hline $\begin{array}{l}\text { Farias Júnior } \\
17 / 2008\end{array}$ & $\begin{array}{c}\text { João } \\
\text { Pessoa/PB }\end{array}$ & $\begin{array}{l}\text { Schoolchildren } \\
\text { (secondary } \\
\text { school) }\end{array}$ & 2,566 & $14-18$ & $\begin{array}{c}\text { Daily } \\
\text { physical } \\
\text { activity log }\end{array}$ & All & $\begin{array}{l}\text { Physical } \\
\text { inactivity }\end{array}$ & $\begin{array}{l}\text { Energy expenditure } \\
<37 \mathrm{kcal} / \mathrm{kg} / \text { day }\end{array}$ & $\begin{array}{l}\text { Overall: } 55.9 \% \text { (boys: } \\
\text { 45.5\%; girls: } 64.2 \% \text { ) }\end{array}$ \\
\hline $\begin{array}{l}\text { Rodrigues et } \\
\text { al. } 18 / 2008\end{array}$ & Gurupi/TO & $\begin{array}{l}\text { Schoolchildren } \\
\text { (university } \\
\text { students) }\end{array}$ & 871 & $\begin{array}{l}16-57 \\
\text { (mean } \\
=25 \text { ) }\end{array}$ & $\begin{array}{l}\text { IPAQ - short } \\
\text { version }\end{array}$ & $\begin{array}{l}\text { Leisure, work, } \\
\text { household } \\
\text { and commuting }\end{array}$ & $\begin{array}{l}\text { Sedentary } \\
\text { lifestyle } \\
\end{array}$ & Not mentioned & Overall: $29.9 \%$ \\
\hline \multirow[t]{2}{*}{$\begin{array}{l}\text { Siqueira et } \\
\text { al. } 13 / 2008\end{array}$} & \multirow[t]{2}{*}{$\begin{array}{l}\text { South and } \\
\text { Northeast } \\
\text { of Brazil }\end{array}$} & \multirow[t]{2}{*}{$\begin{array}{l}\text { Population } \\
\text { (coverage areas } \\
\text { for primary } \\
\text { healthcare units) }\end{array}$} & 4,060 & $30-64$ & \multirow[t]{2}{*}{$\begin{array}{l}\text { IPAQ - short } \\
\text { version }\end{array}$} & \multirow[t]{2}{*}{$\begin{array}{l}\text { Leisure, work, } \\
\text { household and } \\
\text { commuting }\end{array}$} & \multirow[t]{2}{*}{$\begin{array}{l}\text { Sedentary } \\
\text { lifestyle }\end{array}$} & \multirow[t]{2}{*}{$\begin{array}{c}<150 \text { minutes/week of } \\
\text { moderate to vigorous } \\
\text { physical activity }\end{array}$} & $\begin{array}{l}\text { Adults (overall: } \\
31.8 \% \text {; men: } 37.5 \% \\
\text { women: } 27.2 \% \text { ) }\end{array}$ \\
\hline & & & 4,003 & $\geq 65$ & & & & & $\begin{array}{c}\text { Elderly (overall: } \\
58.0 \% \text {; men: } 59.6 \% \text {; } \\
\text { women: } 56.9 \% \text { ) }\end{array}$ \\
\hline
\end{tabular}

States of Brazil: BA: Bahia; GO: Goiás; PB: Paraíba; RJ: Rio de Janeiro; RS: Rio Grande do Sul; SC: Santa Catarina; SP: São Paulo; TO: Tocantins. IPAQ: International Physical Activity Questionnaire. 
Table 2

Studies in which prevalence of physical activity and/or inactivity was assessed as a secondary variable $(n=21)$.

\begin{tabular}{|c|c|c|c|c|c|c|c|c|c|}
\hline Author/Year & City/State & Sample Type & $\mathbf{N}$ & $\begin{array}{c}\text { Age } \\
\text { bracket } \\
\text { (years) }\end{array}$ & $\begin{array}{l}\text { Instru- } \\
\text { ment }\end{array}$ & $\begin{array}{l}\text { Domain of } \\
\text { physical } \\
\text { activity }\end{array}$ & $\begin{array}{l}\text { Study } \\
\text { variable }\end{array}$ & Definition & Prevalence \\
\hline $\begin{array}{l}\text { Rego et al. } \\
9 / 1990\end{array}$ & $\begin{array}{l}\text { São Paulo/ } \\
\text { SP }\end{array}$ & Population & 1,914 & $15-59$ & $\begin{array}{l}\text { Own } \\
\text { question- } \\
\text { naire. }\end{array}$ & $\begin{array}{l}\text { Not } \\
\text { mentioned }\end{array}$ & $\begin{array}{l}\text { Sedentary } \\
\text { lifestyle }\end{array}$ & Not mentioned & $\begin{array}{l}\text { Overall: } 69.3 \% \text { (men: } \\
57.3 \% \text {; women: } 80.2 \% \text { ) }\end{array}$ \\
\hline $\begin{array}{l}\text { Duncan et al. } \\
10 / 1993\end{array}$ & $\begin{array}{c}\text { Porto } \\
\text { Alegre/RS }\end{array}$ & Population & 1,240 & $15-64$ & $\begin{array}{l}\text { Own } \\
\text { question- } \\
\text { naire }\end{array}$ & $\begin{array}{l}\text { Leisure, work, } \\
\text { and commuting }\end{array}$ & $\begin{array}{l}\text { Sedentary } \\
\text { lifestyle }\end{array}$ & $\begin{array}{l}\text { Sedentary work and } \\
\text { commuting }<30 \\
\text { minutes/day and } \\
\text { leisure-time physical } \\
\text { activity }<4 \text { hours/week } \\
\text { (moderate) or } \\
<3 \text { hours/ } \\
\text { week (vigorous) }\end{array}$ & $\begin{array}{l}\text { Overall (men: } 38.0 \% \\
\text { women: } 58.0 \% \text { ) } \\
\text { Leisure (men: } 69.0 \% \\
\text { women: } 82.0 \% \text { ) }\end{array}$ \\
\hline $\begin{array}{l}\text { Barreto et al. } \\
57 / 2001\end{array}$ & Bambuí/MG & Population & 2,284 & $\geq 15$ & $\begin{array}{l}\text { Own } \\
\text { question- } \\
\text { naire }\end{array}$ & Not mentioned & $\begin{array}{l}\text { Physical } \\
\text { inactivity }\end{array}$ & $\begin{array}{c}\text { No weekly physical } \\
\text { activity }\end{array}$ & Overall: $26.7 \%$ \\
\hline \multirow{2}{*}{$\begin{array}{l}\text { Barros } \\
\& \text { Nahas } \\
28 / 2001\end{array}$} & \multirow[t]{2}{*}{$\begin{array}{c}\text { Santa } \\
\text { Catarina/SC }\end{array}$} & \multirow[t]{2}{*}{$\begin{array}{l}\text { Workers } \\
\text { (industrial) }\end{array}$} & \multirow[t]{2}{*}{4,850} & \multirow[t]{2}{*}{$18-71$} & \multirow[t]{2}{*}{$\begin{array}{l}\text { IPAQ - long } \\
\text { version }\end{array}$} & \multirow[t]{2}{*}{ Leisure } & $\begin{array}{l}\text { Physical } \\
\text { inactivity }\end{array}$ & $\begin{array}{c}\text { No weekly physical } \\
\text { activity }\end{array}$ & $\begin{array}{l}\text { Overall: } 46.2 \% \text { (men: } \\
34.8 \% \text {; women: } 67.0 \% \text { ) }\end{array}$ \\
\hline & & & & & & & $\begin{array}{l}\text { Insufficient } \\
\text { physical } \\
\text { activity }\end{array}$ & $\begin{array}{l}\text { Energy expenditure } \\
\text { in physical activity } \\
<14,3 \mathrm{kcal} / \mathrm{kg} / \text { week }\end{array}$ & $\begin{array}{l}\text { Overall: } 21.4 \% \text { (men: } \\
24.2 \% \text {; women: } 16.4 \% \text { ) }\end{array}$ \\
\hline \multirow[t]{2}{*}{$\begin{array}{l}\text { Lima-Costa } \\
\text { et al. } 58 / 2001\end{array}$} & \multirow[t]{2}{*}{ Bambuí/MG } & \multirow[t]{2}{*}{ Population } & 1,020 & $18-59$ & \multirow[t]{2}{*}{$\begin{array}{l}\text { Own } \\
\text { question- } \\
\text { naire }\end{array}$} & \multirow[t]{2}{*}{ Leisure } & $\begin{array}{l}\text { Physical } \\
\text { inactivity }\end{array}$ & $\begin{array}{c}\text { No day of physical } \\
\text { activity ( } \geq 30 \text { minutes) in } \\
\text { previous month }\end{array}$ & $\begin{array}{c}\text { Adults: } 67.2 \% \text {; elderly: } \\
79.5 \%\end{array}$ \\
\hline & & & 1,606 & $\geq 60$ & & & $\begin{array}{l}\text { Sedentary } \\
\text { lifestyle }\end{array}$ & $\begin{array}{l}\text { Spends most of the day } \\
\text { sitting and walks little }\end{array}$ & $\begin{array}{c}\text { Adults: } 28.0 \% \text {; elderly: } \\
47.7 \%\end{array}$ \\
\hline $\begin{array}{l}\text { Gus et al. } \\
59 / 2002\end{array}$ & $\begin{array}{l}\text { Rio Grande } \\
\text { do Sul/RS }\end{array}$ & Population & 1,066 & $\geq 20$ & $\begin{array}{l}\text { Own } \\
\text { question- } \\
\text { naire }\end{array}$ & $\begin{array}{l}\text { Leisure and } \\
\text { work }\end{array}$ & $\begin{array}{l}\text { Sedentary } \\
\text { lifestyle }\end{array}$ & $\begin{array}{l}\text { Light physical activity } \\
<2 \text { days/weeks }\end{array}$ & Overall: $71.3 \%$ \\
\hline $\begin{array}{l}\text { Matos \& } \\
\text { Ladeia } \\
30 / 2003\end{array}$ & $\begin{array}{l}\text { Ipecaetá/ } \\
\text { BA }\end{array}$ & $\begin{array}{l}\text { Population (rural } \\
\text { community) }\end{array}$ & 160 & $\geq 19$ & $\begin{array}{l}\text { Own } \\
\text { question- } \\
\text { naire }\end{array}$ & $\begin{array}{l}\text { Leisure and } \\
\text { work }\end{array}$ & $\begin{array}{l}\text { Physical } \\
\text { inactivity }\end{array}$ & $\begin{array}{c}\text { Physical activity } \\
<2 \text { days/week or } \\
\text { low energy expenditure } \\
\text { at week }\end{array}$ & Overall: $43.5 \%$ \\
\hline $\begin{array}{l}\text { Farias Júnior } \\
\text { \& Lopes } \\
\text { 19/2004 }\end{array}$ & $\begin{array}{l}\text { Florianó- } \\
\text { polis/SC }\end{array}$ & $\begin{array}{l}\text { Schoolchildren } \\
\text { (secondary } \\
\text { school) }\end{array}$ & 1,107 & $15-18$ & $\begin{array}{l}\text { Own } \\
\text { question- } \\
\text { naire }\end{array}$ & All & $\begin{array}{l}\text { Insufficient } \\
\text { physical } \\
\text { activity }\end{array}$ & $\begin{array}{l}\text { Energy expenditure } \\
<37 \mathrm{kcal} / \mathrm{kg} / \text { day }\end{array}$ & $\begin{array}{l}\text { Overall: } 65.7 \% \text { (boys: } \\
52.1 \% \text {; girls: } 78.3 \% \text { ) }\end{array}$ \\
\hline $\begin{array}{l}\text { Lima-Costa } \\
60 / 2004\end{array}$ & $\begin{array}{l}\text { Belo } \\
\text { Horizonte/ } \\
\text { MG }\end{array}$ & Population & 13,851 & $\geq 20$ & $\begin{array}{l}\text { Own } \\
\text { question- } \\
\text { naire }\end{array}$ & Leisure & $\begin{array}{l}\text { Physical } \\
\text { activity }\end{array}$ & $\begin{array}{c}\geq 20 \text { minutes/day, daily } \\
\text { or almost daily in the } \\
\text { previous } 3 \text { months }\end{array}$ & $\begin{array}{l}\text { Overall: } 11.2 \% \text { (adults: } \\
\text { 10.8\%; elderly: } 14.0 \% \text { ) }\end{array}$ \\
\hline $\begin{array}{l}\text { Monteiro et } \\
\text { al. } 39 / 2005\end{array}$ & $\begin{array}{l}\text { São Paulo/ } \\
\text { SP }\end{array}$ & Population & 2,122 & $\geq 18$ & $\begin{array}{l}\text { Own } \\
\text { question- } \\
\text { naire } \\
\text { (telephone } \\
\text { interviews) }\end{array}$ & Leisure & $\begin{array}{l}\text { Physical } \\
\text { activity }\end{array}$ & $\begin{array}{c}\text { None } \\
\geq \text { once/week } \\
\geq 3 \text { times/week } \\
\geq 5 \text { times/week }\end{array}$ & $\begin{array}{c}47.0 \% \\
29.0 \% \\
16.5 \% \\
6.5 \%\end{array}$ \\
\hline $\begin{array}{l}\text { Silva et al. } \\
20 / 2005\end{array}$ & Maceió/AL & Schoolchildren & 1,253 & $7-17$ & PAQ & $\begin{array}{l}\text { Leisure and } \\
\text { school }\end{array}$ & $\begin{array}{l}\text { Sedentary } \\
\text { lifestyle }\end{array}$ & Not mentioned (scores) & $\begin{array}{l}\text { Overall: 93.5\% (boys: } \\
90.3 \% \text {; girls: } 96.0 \%)\end{array}$ \\
\hline \multirow[t]{2}{*}{$\begin{array}{l}\text { Conceição et } \\
\text { al. } 29 / 2006\end{array}$} & \multirow[t]{2}{*}{$\begin{array}{c}\text { João } \\
\text { Pessoa/PB }\end{array}$} & \multirow{2}{*}{$\begin{array}{l}\text { Workers } \\
\text { (university } \\
\text { employees) }\end{array}$} & \multirow[t]{2}{*}{704} & \multirow[t]{2}{*}{$40-72$} & \multirow{2}{*}{$\begin{array}{l}\text { Own } \\
\text { question- } \\
\text { naire }\end{array}$} & \multirow[t]{2}{*}{$\begin{array}{l}\text { Not } \\
\text { mentioned }\end{array}$} & $\begin{array}{l}\text { Sedentary } \\
\text { lifestyle }\end{array}$ & No physical activity & $\begin{array}{l}\text { Overall: } 48.4 \% \text { (men: } \\
45.4 \% \text {; women: } 53.0 \% \text { ) }\end{array}$ \\
\hline & & & & & & & $\begin{array}{l}\text { Regular } \\
\text { physical } \\
\text { activity }\end{array}$ & $\begin{array}{c}\geq 30 \text { minutes at least } 4 \\
\text { days/week }\end{array}$ & Overall: $20.1 \%$ \\
\hline $\begin{array}{l}\text { Doro et al. } \\
61 / 2006\end{array}$ & Bauru/SP & $\begin{array}{l}\text { Japanese } \\
\text { descendants }\end{array}$ & 1,330 & $\geq 30$ & $\begin{array}{l}\text { Own } \\
\text { question- } \\
\text { naire }\end{array}$ & $\begin{array}{l}\text { Leisure and } \\
\text { work }\end{array}$ & $\begin{array}{l}\text { Physical } \\
\text { activity }\end{array}$ & Not defined & $\begin{array}{c}\text { Men: } 18.8 \% \\
\text { women: } 13.4 \%\end{array}$ \\
\hline $\begin{array}{l}\text { Monego } \\
\text { \& Jardim } \\
21 / 2006\end{array}$ & Goiânia/GO & Schoolchildren & 3,169 & $7-14$ & $\begin{array}{l}\text { Own } \\
\text { question- } \\
\text { naire }\end{array}$ & $\begin{array}{l}\text { Leisure and } \\
\text { school }\end{array}$ & $\begin{array}{l}\text { Sedentary } \\
\text { lifestyle }\end{array}$ & $\begin{array}{l}\text { No physical activity } \\
\text { most of the time }\end{array}$ & $\begin{array}{l}\text { Overall: } 37.8 \% \text { (boys: } \\
28.6 \% \text {; girls: } 47.3 \% \text { ) }\end{array}$ \\
\hline
\end{tabular}


Table 2 (continued)

\begin{tabular}{|c|c|c|c|c|c|c|c|c|c|}
\hline $\begin{array}{l}\text { Author/ } \\
\text { Year }\end{array}$ & City/State & Sample type & $\mathbf{N}$ & $\begin{array}{l}\text { Age } \\
\text { bracket } \\
\text { (years) }\end{array}$ & $\begin{array}{l}\text { Instru- } \\
\text { ment }\end{array}$ & $\begin{array}{l}\text { Domain of } \\
\text { physical } \\
\text { activity }\end{array}$ & $\begin{array}{c}\text { Study } \\
\text { variable }\end{array}$ & Definition & Prevalence \\
\hline $\begin{array}{l}\text { Nobre et al. } \\
22 / 2006\end{array}$ & $\begin{array}{l}\text { São Paulo/ } \\
\text { SP }\end{array}$ & $\begin{array}{l}\text { Schoolchildren } \\
\text { (5th to } 8^{\text {th }} \text { grades, } \\
\text { primary school) }\end{array}$ & 2,125 & $\begin{array}{c}\text { Not } \\
\text { defined }\end{array}$ & $\begin{array}{l}\text { Adapted } \\
\text { from } \\
\text { question- } \\
\text { naire used } \\
\text { in "Carmen- } \\
\text { Cindi" } \\
\text { (1997) }\end{array}$ & Not mentioned & $\begin{array}{l}\text { Sedentary } \\
\text { lifestyle }\end{array}$ & $\begin{array}{l}\text { Walks very little or } \\
\text { spends most of } \\
\text { the day sitting }\end{array}$ & Overall: $15.4 \%$ \\
\hline \multirow[t]{4}{*}{$\begin{array}{l}\text { Carvalhaes } \\
\text { et al. } 40 / 2008\end{array}$} & \multirow[t]{4}{*}{8} & \multirow[t]{4}{*}{ Population } & \multirow[t]{4}{*}{1,410} & \multirow[t]{4}{*}{$\geq 18$} & \multirow{4}{*}{$\begin{array}{l}\text { Own } \\
\text { question- } \\
\text { naire } \\
\text { (telephone } \\
\text { interviews) }\end{array}$} & \multirow{4}{*}{$\begin{array}{l}\text { Leisure and } \\
\text { work }\end{array}$} & $\begin{array}{l}\text { Sedentary } \\
\text { lifestyle }\end{array}$ & $\begin{array}{l}\text { No leisure-time physi- } \\
\text { cal activity and light } \\
\text { effort at work }\end{array}$ & $\begin{array}{l}\text { Overall: } 57.9 \% \text { (men: } \\
\text { 43.1\%; women: } 58.2 \% \text { ) }\end{array}$ \\
\hline & & & & & & & $\begin{array}{l}\text { Leisure- } \\
\text { time } \\
\text { physical } \\
\text { activity (1) }\end{array}$ & $\begin{array}{c}30 \text { minutes at least } 1 \\
\text { day/week }\end{array}$ & $\begin{array}{l}\text { Overall: } 31.0 \% \text { (men: } \\
\text { 36.9\%; women: } 25.8 \% \text { ) }\end{array}$ \\
\hline & & & & & & & $\begin{array}{l}\text { Leisure- } \\
\text { time } \\
\text { physical } \\
\text { activity (2) }\end{array}$ & $\begin{array}{c}30 \text { minutes at least } 3 \\
\text { days/week }\end{array}$ & $\begin{array}{l}\text { Overall: } 26.6 \% \text { (men: } \\
22.2 \% \text {; women: } 19.3 \% \text { ) }\end{array}$ \\
\hline & & & & & & & $\begin{array}{l}\text { Leisure- } \\
\text { time } \\
\text { physical } \\
\text { activity (3) }\end{array}$ & $\begin{array}{c}30 \text { minutes at least } 5 \\
\text { days/week }\end{array}$ & $\begin{array}{l}\text { Overall: } 12.1 \% \text { (men: } \\
\text { 12.7\%; women: } 9.0 \% \text { ) }\end{array}$ \\
\hline \multirow[t]{5}{*}{$\begin{array}{l}\text { Castro et al. } \\
23 / 2008\end{array}$} & \multirow[t]{5}{*}{$\begin{array}{c}\text { Rio de } \\
\text { Janeiro/RJ }\end{array}$} & \multirow{5}{*}{$\begin{array}{l}\text { Schoolchildren } \\
\text { (8th grade, } \\
\text { primary school) }\end{array}$} & \multirow[t]{5}{*}{1,684} & \multirow{5}{*}{$\begin{array}{c}\geq 13 \\
\text { (median }= \\
15 \text { years) }\end{array}$} & \multirow{5}{*}{$\begin{array}{l}\text { Own } \\
\text { question- } \\
\text { naire }\end{array}$} & \multirow[t]{5}{*}{$\begin{array}{l}\text { Leisure and } \\
\text { commuting }\end{array}$} & $\begin{array}{l}\text { Physical } \\
\text { inactivity }\end{array}$ & No physical activity & $\begin{array}{l}\text { Overall: } 9.7 \% \text { (boys: } \\
5.0 \% \text {; girls: } 11.9 \% \text { ) }\end{array}$ \\
\hline & & & & & & & $\begin{array}{l}\text { Physical } \\
\text { activity } 1\end{array}$ & $\begin{array}{c}60 \text { minutes } 3 \text { days/ } \\
\text { week }\end{array}$ & $\begin{array}{l}\text { Overall: } 37.1 \% \text { (boys: } \\
55.6 \% \text {; girls: } 20.0 \% \text { ) }\end{array}$ \\
\hline & & & & & & & $\begin{array}{l}\text { Physical } \\
\text { activity } 2\end{array}$ & $\begin{array}{c}60 \text { minutes } 5 \text { days/ } \\
\text { week }\end{array}$ & $\begin{array}{l}\text { Overall: } 18.7 \% \text { (boys: } \\
\text { 29.8\%; girls: } 8.3 \% \text { ) }\end{array}$ \\
\hline & & & & & & & $\begin{array}{l}\text { Physical } \\
\text { activity } 3\end{array}$ & $\begin{array}{l}150 \text { minutes/ } \\
\text { week }\end{array}$ & $\begin{array}{l}\text { Overall: } 59.9 \% \text { (boys: } \\
73.6 \% \text {; girls: } 47.2 \% \text { ) }\end{array}$ \\
\hline & & & & & & & $\begin{array}{l}\text { Physical } \\
\text { activity } 4\end{array}$ & $\begin{array}{l}300 \text { minutes/ } \\
\text { week }\end{array}$ & $\begin{array}{l}\text { Overall: } 40.1 \% \text { (boys: } \\
55.2 \% \text {; girls: } 26.1 \% \text { ) }\end{array}$ \\
\hline \multirow[t]{2}{*}{$\begin{array}{l}\text { Moura et } \\
\text { al. } 8 / 2008\end{array}$} & \multirow{2}{*}{$\begin{array}{l}26 \text { State } \\
\text { capitals }+ \\
\text { Federal } \\
\text { District }\end{array}$} & \multirow[t]{2}{*}{ Population } & \multirow[t]{2}{*}{54,369} & \multirow[t]{2}{*}{$\geq 18$} & \multirow{2}{*}{$\begin{array}{l}\text { Not } \\
\text { mentioned } \\
\text { (telephone } \\
\text { interviews) }\end{array}$} & \multirow{2}{*}{$\begin{array}{l}\text { Leisure, work, } \\
\text { household and } \\
\text { commuting }\end{array}$} & $\begin{array}{l}\text { Sedentary } \\
\text { lifestyle }\end{array}$ & No physical activity & $\begin{array}{l}\text { Overall: } 29.2 \% \text { (men: } \\
\text { 39.8\%; women: } 20.1 \% \text { ) }\end{array}$ \\
\hline & & & & & & & $\begin{array}{l}\text { Sufficient } \\
\text { leisure- } \\
\text { time } \\
\text { physical } \\
\text { activity }\end{array}$ & $\begin{array}{l}30 \text { minutes/day of } \\
\text { moderate physical } \\
\text { activity } \geq 5 \text { times/week } \\
\text { or } 20 \text { minutes/day } \\
\text { of vigorous physical } \\
\text { activity } \geq 3 \text { times/week }\end{array}$ & $\begin{array}{l}\text { Overall: } 14.9 \% \text { (men: } \\
\text { 18.3\%; women: } 11.9 \%)\end{array}$ \\
\hline $\begin{array}{l}\text { Peixoto et } \\
\text { al. } 41 / 2008\end{array}$ & Goiânia/GO & Population & 2,002 & $\geq 18$ & $\begin{array}{l}\text { Own } \\
\text { question- } \\
\text { naire } \\
\text { (telephone } \\
\text { interviews) }\end{array}$ & $\begin{array}{l}\text { Leisure, work, } \\
\text { household and } \\
\text { commuting }\end{array}$ & $\begin{array}{l}\text { Sedentary } \\
\text { lifestyle }\end{array}$ & $\begin{array}{l}\text { No leisure-time, } \\
\text { commuting, or } \\
\text { household physical } \\
\text { activity and light } \\
\text { effort at work }\end{array}$ & $\begin{array}{l}\text { Overall: } 29.7 \% \text { (men: } \\
34.8 \% \text {; women: } 36.6 \% \text { ) }\end{array}$ \\
\hline $\begin{array}{l}\text { Romanzini et } \\
\text { al. } 24 / 2008\end{array}$ & Londrina/PR & $\begin{array}{l}\text { Schoolchildren } \\
\text { (secondary } \\
\text { school) }\end{array}$ & 644 & $14-19$ & $\begin{array}{l}\text { IPAQ - short } \\
\text { version }\end{array}$ & $\begin{array}{l}\text { Leisure, work, } \\
\text { household and } \\
\text { commuting }\end{array}$ & $\begin{array}{l}\text { Physical } \\
\text { inactivity }\end{array}$ & $\begin{array}{c}<300 \text { minutes/week of } \\
\text { moderate to vigorous } \\
\text { physical activity }\end{array}$ & $\begin{array}{l}\text { Overall: } 39.2 \% \text { (boys: } \\
\text { 33.3\%; girls: } 42.8 \% \text { ) }\end{array}$ \\
\hline $\begin{array}{l}\text { Silva et al. } \\
25 / 2008\end{array}$ & $\begin{array}{c}\text { Santa } \\
\text { Catarina/SC }\end{array}$ & $\begin{array}{l}\text { Schoolchildren } \\
\text { (secondary } \\
\text { school) }\end{array}$ & 5,024 & $15-19$ & $\begin{array}{l}\text { Own } \\
\text { question- } \\
\text { naire }\end{array}$ & $\begin{array}{l}\text { Leisure, work, } \\
\text { household and } \\
\text { commuting }\end{array}$ & $\begin{array}{l}\text { Limited } \\
\text { activity }\end{array}$ & $\begin{array}{c}<300 \text { minutes/week of } \\
\text { moderate to vigorous } \\
\text { physical activity }\end{array}$ & $\begin{array}{l}\text { Overall: } 31.0 \% \text { (boys: } \\
21.0 \% \text {; girls: } 37.0 \% \text { ) }\end{array}$ \\
\hline
\end{tabular}

States of Brazil: AL: Alagoas; BA: Bahia; GO: Goiás; MG: Minas Gerais; PB: Paraíba; PR: Paraná; RJ: Rio de Janeiro; RS: Rio Grande do Sul; SC: Santa Catarina; SP: São Paulo.

IPAQ: International Physical Activity Questionnaire; PAQ: Physical Activity Questionnaire. 


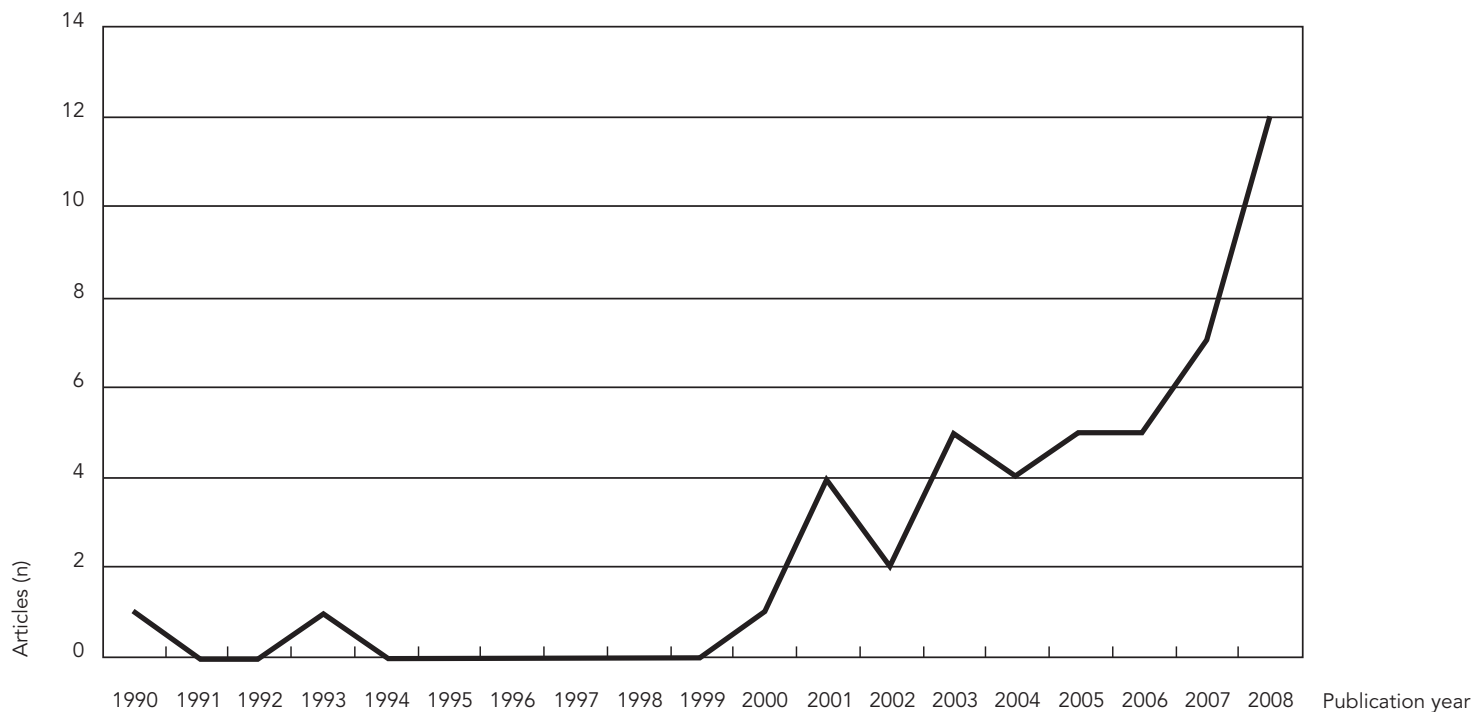

more included cities in more than one State or region 12,13. The South of Brazil had the most articles (22), followed by the Southeast (16), Northeast (8), Central-West (4), and North (2). The first study with data from the North of Brazil was only published in 2008.

The vast majority of the study samples were population-based (33), and two studies only included women 14,15 . In ten studies, the sample was school-based 16,17,18,19,20,21,22,23,24,25 and four included workers $26,27,28,29$. The sample size ranged from 160 individuals 30 to 54,369 8, while the majority of the studies (38) included more than a thousand subjects, and only two included fewer than five hundred 30,31.

As for age bracket, some two-thirds of the studies (32) included adults (18-59 years) and more than half (28) included elderly ( $\geq 60$ years), while in two articles the study sample included only elderly individuals 31,32 . In all, 20 studies included adolescents (10-17 years), of which tem included only this age bracket. Only two studies included children (7-9 years) in the sample 20,21, neither of which exclusively.

In all the studies, physical activity was measured subjectively, i.e., based on subjects' selfreported information. Questionnaires were the most widely used instrument (45 studies). Although the majority of the studies used their own questionnaires, ten used the Inter- national Physical Activity Questionnaire (IPAQ) $13,18,24,28,32,33,34,35,36,37$. In five studies the interviews were conducted by telephone $8,38,39,40,41$. Two studies used daily logs to measure physical activity 16,17 .

As for domain, 14 studies only investigated leisure-time physical activity, while 13 analyzed total physical activity, i.e., including leisure-time, work, commuting, and household. The most widely studied behavior was sedentary lifestyle (22 studies). Twenty articles assessed prevalence of physical activity, and 16 focused on physical inactivity with its variations (e.g., insufficient physical activity), not counting those that used the nomenclature "sedentary". Figure 2 summarizes the terminology used.

The widest diversity among the studies relates to the criteria for defining the target behaviors (physical activity, inactivity, sedentary lifestyle, and others). Still, as shown in Tables 1 and 2 , the most common criterion used with adults and the elderly is 150 minutes per week of moderate to vigorous activity, found in eight studies $8,12,13,32,33,34,36,40$. The most common criterion for active children and adolescents was at least 300 minutes of physical activity per week, found in six articles 23,24,25,42,43,44.

Finally, prevalence of physical activity also varied widely among the studies, from $3.3 \% 12$ to $85.4 \% 42$, and was nearly always higher for men 


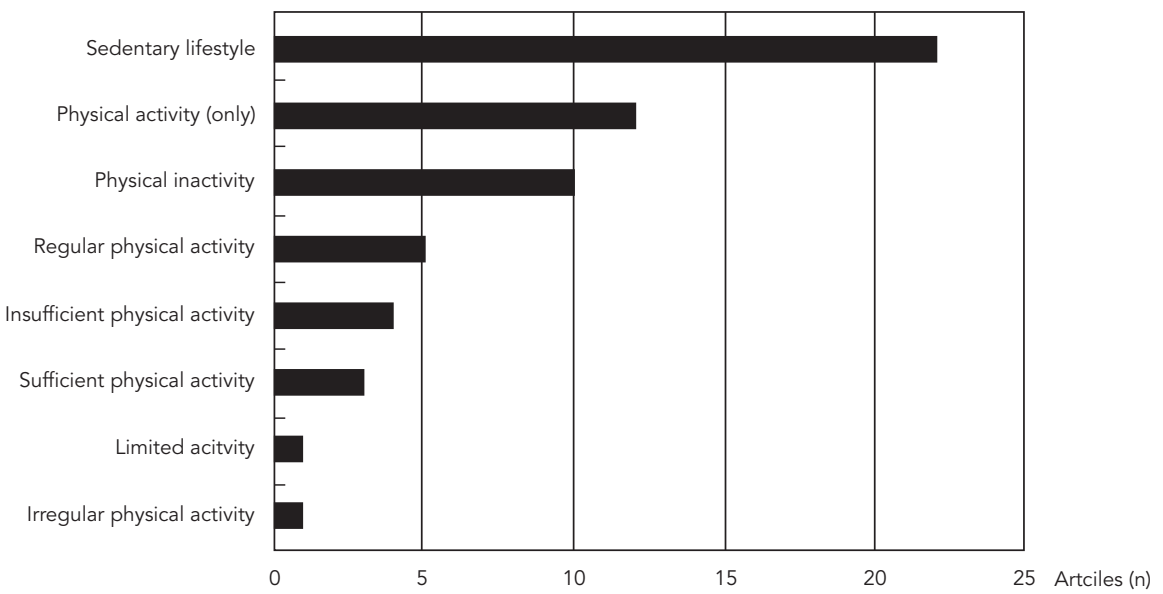

than women. Although not shown in the tables, there is an inverse association between level of physical activity and age, i.e., the older the individual, the lower the prevalence of physical activity.

\section{Discussion}

The current study aimed to systematically compile all the Brazilian studies containing data on prevalence of physical activity or inactivity in representative samples of the population. This is the first (and thus far the only) systematic review in Brazil with this approach, although two other articles have described the epidemiological studies on physical activity, but without approaching the results in terms of prevalence 1,45 .

Like any other study, this one is also subject to some limitations. Although the literature search was conducted carefully, some article that met the inclusion criteria may not have been identified. This could have happened for two main reasons: (1) physical activity was a secondary variable, i.e., examined jointly with a set of other variables, and the article was not identified in the search and (2) the article is not indexed in any of the databases that were searched, nor does it appear in Google Scholar. However, even though some article may not have been included, it is believed that with the methodology used, the main studies related to the theme were identified.
The review only included studies with probabilistic samples, because convenience samples may not reflect a given behavior's occurrence and/or distribution in the target population. Thus, the review excluded all the studies with non-random samples. Importantly, however, no limit was set on sample size. For example, some studies had fewer than 500 individuals, although the vast majority included more than one thousand each.

Additionally, the literature search placed no restrictions as to date of publication or language. This allowed drawing a timeline for the articles, starting with the first in 1990 to the most recent in 2008. Year of publication was chosen rather than year of data collection, because most studies did not provide the latter information. In addition, studies published in English were included (both from Brazilian and foreign journals). Although publication of Brazilian articles in English is not common, it has increased in recent years, thereby expanding access by foreign researchers to studies conducted in Brazil. The review's main findings are highlighted in the following paragraphs.

First, there was a substantial increase in articles on physical activity in recent years. Of all the studies identified, only two were published before 2000, while 12 articles were published in 2008 alone. Except for three studies (one nationwide, one in the South and Northeast of Brazil, and one in the Southeast and Northeast), the others were conducted in a single State or city. There was a wide disparity of publications between the 
geographic regions of Brazil. For example, the city of Pelotas alone (in Rio Grande do Sul State) had ten articles included in this review (some 20\% of the total). There was a major concentration of studies in the South and Southeast regions, with the majority of the publications, and few in the Central-West, North, and Northeast. In the North, for example, no study had been published as of 2007.

Distribution by age bracket shows few studies including children (only two were identified). This may have been due to logistic difficulties in collecting data from this age group. There were also few studies done exclusively on the elderly, although several studies included this age group together with adults in general.

All the studies adopted a cross-sectional design. Although this design is appropriate for investigating a given event's occurrence in the population, oftentimes it does not allow establishing temporal relations in the target associations or changes over time. Therefore, cohort studies are needed to study trends in physical activity over time in a given group of individuals, as well as factors that determine variation in physical activity during different stages of life.

As for the instruments used to measure physical activity, the review showed a strong preference for questionnaires, probably for cost-benefit reasons. Nevertheless, the studies adopted a wide range of instruments. The vast majority of authors applied their own questionnaires and/ or those adapted (but not validated) from others. This does not rule out the quality of such studies, but does hinder comparison of the results with those of other studies. The most widely used instrument was the IPAQ (short version), which investigates various domains of physical activity. This questionnaire's validity and reproducibility have been tested in 12 countries, including Brazil 46. The Global Physical Activity Questionnaire (GPAQ) 47 , an adaptation of the IPAQ developed by the WHO, is another questionnaire that has been used at the international level to measure level of physical activity.

Only two studies used daily logs (both of which with adolescents). In the coming years, studies may emerge that measure physical activity objectively, especially using pedometers and accelerometers, although such instruments also have their limitations. This practice has been adopted in developed countries, including at the population level 48 . Concerning domain of physical activity, while the first studies focused only on leisure time, more recent studies have emphasized physical activity in the different domains (leisure, work, commuting, and household).
The recall period for physical activity also varied between studies (the tables do not contain this information, since several authors failed to mention it). Among the articles that provided this information, there was a balance between recall during the previous week and during a normal week. An alternative used in international studies has been to collect both pieces of information and analyze the mean for the two (previous week and normal week) 49 .

The current study clearly shows that besides a wide range of instruments, there is no consensus as to the terminology for either describing the target behavior (e.g., sedentary lifestyle, physical inactivity, insufficient physical activity) or the criteria adopted to define such behaviors. Thus, there are authors studying the same behavior but with different nomenclatures, as well as different behaviors with the same nomenclature. This emphasizes the urgent need to standardize the terminology as well as the criteria for defining each behavior. Meanwhile, especially in studies with physical activity as the main variable, there is a trend to study prevalence of physical activity rather than physical inactivity (or sedentary lifestyle), as was done preferentially in the older studies.

With regard to recommendations for physical activity, despite the lack of international consensus, the most widely adopted guidelines are those endorsed in 2007 by the American College of Sports Medicine (ACSM) and the American Heart Association (AHA). The two associations recommend that all healthy adults (18-65 years of age) practice a minimum of 30 minutes of moderate physical activity at least 5 days a week, or 20 minutes of vigorous activity at least 3 days a week, or a combination of the two 50 . Such activities need not be continuous, but they should last a minimum of 10 minutes each, according to the guidelines. For children and adolescents, the same authors refer to a previous publication in which 850 articles were reviewed, reaching the conclusion that school-age youngsters (6-18 years) should practice at least 60 minutes of moderate to vigorous physical activity every day of the week 51. However, some countries (e.g., Canada, England, and Australia) have specific agencies dealing with the subject, and the guidelines are not always consistent with those mentioned above, and can even vary within the same country. A recent example appears in the new guidelines published by the United States Department of Health and Human Services (USDHHS) 2 in 2008, recommending that individuals 18 years or older practice at least $150 \mathrm{~min}$ utes a week of moderate-intensity aerobic activity or 75 minutes a week of vigorous activity. 
Thus, all these differences (instruments, recall, domains, terminology, and criteria) make it impossible to compare the different studies' results for prevalence of physical activity. However, an almost universal finding is that physical activity is more prevalent in males and tends to decrease with age (cross-sectional data).

In conclusion, despite the inability to compare the prevalence of physical activity (or inactivity) in Brazilian studies, the attempt was to present each study's results according to its main methodological aspects. This approach allowed identifying certain limitations and gaps that need to be overcome or filled in future research. It is hoped that the current review will serve as a reference for students, professors, and researchers in the field of physical activity and health, not as a definitive position, but as a point of departure for subsequent work. The aim was also to contribute to the enhancement of this field of knowledge and encourage publication of new studies focusing on this important issue.

\section{Resumo}

O objetivo deste estudo foi descrever, por meio de uma revisão sistemática, a prevalência de atividade (ou inatividade) física no Brasil. As bases de dados consultadas foram: LILACS, SciELO, MEDLINE, Web of Science e o portal Google Acadêmico. Os termos de busca foram: "atividade física", "exercício físico", "inatividade física", "sedentarismo", "Brasil” e "brasileiros". Foram localizados 47 estudos (todos transversais) com dados sobre prevalência de atividade física em amostras aleatórias, sendo que em 26, esta foi a principal variável. Apenas dois estudos foram publicados antes de 2000, e 12 em 2008. Houve uma grande concentração de trabalhos nas regiões Sul e Sudeste, e poucos estudos com crianças. Em todos os trabalhos, a atividade física foi mensurada de forma subjetiva, principalmente por meio de questionários, e o domínio mais estudado foi o lazer. Os critérios para definir atividade física variaram amplamente, assim como a sua prevalência. Evidencia-se a necessidade de padronização dos instrumentos, critérios e nomenclatura nos estudos epidemiológicos sobre atividade física.

Atividade Motora; Exercício; Revisão

\section{Acknowledgments}

The author wishes to thank the Brazilian Council for Scientific and Technological Development (CNPq) for providing a PhD scholarship.

\section{References}

1. Hallal PC, Dumith SC, Bastos JP, Reichert FF, Siqueira FV, Azevedo MR. Evolução da pesquisa epidemiológica em atividade física no Brasil: revisão sistemática. Rev Saúde Pública 2007; 41:453-60.

2. United States Department of Health and Human Services. Physical Activity Guidelines Advisory Committee Report, 2008. Washington DC: United States Department of Health and Human Services; 2008.

3. World Health Organization. The World Health Report 2002: reducing risks, promoting healthy life. Geneva: World Health Organization; 2002.

4. Guthold R, Ono T, Strong KL, Chatterji S, Morabia A. Worldwide variability in physical inactivity a 51country survey. Am J Prev Med 2008; 34:486-94.

5. Instituto Nacional de Câncer. Inquérito domiciliar sobre comportamentos de risco e morbidade referida de doenças e agravos não transmissíveis. Rio de Janeiro: Instituto Nacional de Câncer; 2003.

6. Ministério da Saúde. VIGITEL Brasil 2008: vigilância de fatores de risco e proteção para doenças crônicas por inquérito telefônico. Brasília: Ministério da Saúde; 2009.

7. Ministério da Saúde. VIGITEL Brasil 2007: vigilância de fatores de risco e proteção para doenças crônicas por inquérito telefônico. Brasília: Ministério da Saúde; 2007. 
8. Moura EC, Morais Neto OL, Malta DC, Moura L, Silva NN, Bernal R, et al. Vigilância de fatores de risco para doenças crônicas por inquérito telefônico nas capitais dos 26 estados brasileiros e no Distrito Federal (2006). Rev Bras Epidemiol 2008; 11 Suppl 1:20-37.

9. Rego RA, Berardo FA, Rodrigues SS, Oliveira ZM, Oliveira MB, Vasconcellos C, et al. Fatores de risco para doenças crônicas não-transmissíveis: inquérito domiciliar no município de São Paulo, SP (Brasil). Metodologia e resultados preliminares. Rev Saúde Pública 1990; 24:277-85.

10. Duncan BB, Schmidt MI, Polanczyk CA, Homrich CS, Rosa RS, Achutti AC. Fatores de risco para doenças não-transmissíveis em área metropolitana na região sul do Brasil: prevalência e simultaneidade. Rev Saúde Pública 1993; 27:43-8.

11. Mello MT, Fernandez AC, Tufik S. Levantamento epidemiológico da prática de atividade física na cidade de São Paulo. Rev Bras Med Esporte 2000; 6:119-24.

12. Monteiro CA, Conde WL, Matsudo SM, Matsudo VR, Bonsenor IM, Lotufo PA. A descriptive epidemiology of leisure-time physical activity in Brazil, 1996-1997. Rev Panam Salud Pública 2003; 14: 246-54.

13. Siqueira FV, Facchini LA, Piccini RX, Tomasi E, Thume E, Silveira DS, et al. Atividade física em adultos e idosos residentes em áreas de abrangência de unidades básicas de saúde de municípios das regiões Sul e Nordeste do Brasil. Cad Saúde Pública 2008; 24:39-54.

14. Masson CR, Dias-da-Costa JS, Olinto MTA, Meneghel S, Costa CC, Bairros F, et al. Prevalência de sedentarismo nas mulheres adultas da cidade de São Leopoldo, Rio Grande do Sul, Brasil. Cad Saúde Pública 2005; 21:1685-95.

15. Domingues MR, Barros AJ. Leisure-time physical activity during pregnancy in the 2004 Pelotas Birth Cohort Study. Rev Saúde Pública 2007; 41:173-80.

16. Arruda ELM, Lopes AS. Gordura corporal, nível de atividade física e hábitos alimentares de adolescentes da região serrana de Santa Catarina, Brasil. Rev Bras Cineantropom Desempenho Hum 2007; 9:5-11.

17. Farias Júnior JC. Associação entre prevalência de inatividade física e indicadores de condição socioeconômica em adolescentes. Rev Bras Med Esporte 2008 ; 14:109-14.

18. Rodrigues ES, Cheik NC, Mayer AF. Nível de atividade física e tabagismo em universitários. Rev Saúde Pública 2008; 42:672-8.

19. Farias Júnior JC, Lopes AS. Comportamentos de risco relacionados à saúde em adolescentes. Rev Bras Ciênc Mov 2004; 12:7-12.

20. Silva MA, Rivera IR, Ferraz MR, Pinheiro AJ, Alves SW, Moura AA, et al. Prevalência de fatores de risco cardiovascular em crianças e adolescentes da rede de ensino da cidade de Maceió. Arq Bras Cardiol 2005; 84:387-92.

21. Monego ET, Jardim PC. Determinantes de risco para doenças cardiovasculares em escolares. Arq Bras Cardiol 2006; 87:37-45.
22. Nobre MRC, Domingues RZL, Silva AR, Colugnati FAB, Taddei JAAC. Prevalências de sobrepeso, obesidade e hábitos de vida associados ao risco cardiovascular em alunos do ensino fundamental. Rev Assoc Med Bras (1992) 2006; 52:118-24.

23. Castro IRR, Cardoso LO, Engstrom EM, Levy RB, Monteiro CA. Vigilância de fatores de risco para doenças não transmissíveis entre adolescentes: a experiência da cidade do Rio de Janeiro, Brasil. Cad Saúde Pública 2008; 24:2279-88.

24. Romanzini M, Reichert FF, Lopes AS, Petroski EL, Farias Júnior JC. Prevalência de fatores de risco cardiovascular em adolescentes. Cad Saúde Pública 2008 ; $24: 2573-81$.

25. Silva KS, Nahas MV, Hoefelmann LP, Lopes AS, Oliveira ES. Associações entre atividade física, índice de massa corporal e comportamentos sedentários em adolescentes. Rev Bras Epidemiol 2008; 11: 159-68.

26. Salles-Costa R, Heilborn ML, Werneck GL, Faerstein E, Lopes CS. Gênero e prática de atividade física de lazer. Cad Saúde Pública 2003; 19 Suppl 2: S325-33.

27. Santos JFS, Coelho CW. Atividade física e obesidade em trabalhadores da indústria. Lecturas, Educación Física y Deportes, Revista Digital 2003; 9(67). http://www.efdeportes.com/efd67/obesidad.htm.

28. Barros MV, Nahas MV. Comportamentos de risco, auto-avaliação do nível de saúde e percepção de estresse entre trabalhadores da indústria. Rev Saúde Pública 2001; 35:554-63.

29. Conceição TV, Gomes FA, Tauil PL, Rosa TT. Valores de pressão arterial e suas associações com fatores de risco cardiovasculares em servidores da Universidade de Brasília. Arq Bras Cardiol 2006; 86:26-31.

30. Matos AC, Ladeia AM. Assessment of cardiovascular risk factors in a rural community in the Brazilian state of Bahia. Arq Bras Cardiol 2003; 81:291-302.

31. Zaitune MPA, Barros MBA, César CLG, Carandina L, Goldbaum M. Fatores associados ao sedentarismo no lazer em idosos, Campinas, São Paulo, Brasil. Cad Saúde Pública 2007; 23:1329-38.

32. Benedetti TR, Borges LJ, Petroski EL, Gonçalves LH. Atividade física e estado de saúde mental de idosos. Rev Saúde Pública 2008; 42:302-7.

33. Matsudo SM, Matsudo VR, Araújo T, Andrade D, Andrade E, Oliveira L, et al. Nível de atividade física da população do Estado de São Paulo: análise de acordo com o gênero, idade, nível sócio-econômico, distribuição geográfica e de conhecimento. Rev Bras Ciênc Mov 2002; 10:41-50.

34. Hallal PC, Victora CG, Wells JC, Lima RC. Physical inactivity: prevalence and associated variables in Brazilian adults. Med Sci Sports Exerc 2003; 35:1894-900.

35. Hallal PC, Siqueira FV. Compliance with vigorous physical activity guidelines in Brazilian adults: prevalence and correlates. J Phys Act Health 2004; 1:389-97.

36. Azevedo MR, Araújo CL, Reichert FF, Siqueira FV, Silva MC, Hallal PC. Gender differences in leisuretime physical activity. Int J Public Health 2007; 52:8-15. 
37. Baretta E, Baretta M, Peres KG. Nível de atividade física e fatores associados em adultos no Município de Joaçaba, Santa Catarina, Brasil. Cad Saúde Pública 2007; 23:1595-602.

38. Cunha IC, Peixoto MRG, Jardim PCBV, Alexandre VP. Fatores associados à prática de atividade física na população adulta de Goiânia: monitoramento por meio de entrevistas telefônicas. Rev Bras Epidemiol 2008; 11:495-504.

40. Carvalhaes M, Moura EC, Monteiro CA. Prevalência de fatores de risco para doenças crônicas: inquérito populacional mediante entrevistas telefônicas em Botucatu, São Paulo, 2004. Rev Bras Epidemiol 2008; 11:14-23.

41. Peixoto MRG, Monego ET, Alexandre VP, Souza RGM, Moura EC. Monitoramento por entrevistas telefônicas de fatores de risco para doenças crônicas: experiência de Goiânia, Goiás, Brasil. Cad Saúde Pública 2008; 24:1323-33.

42. Hallal PC, Bertoldi AD, Gonçalves H, Victora CG. Prevalência de sedentarismo e fatores associados em adolescentes de 10-12 anos de idade. Cad Saúde Pública 2006; 22:1277-87.

43. Gonçalves H, Hallal PC, Amorim TC, Araújo CL, Menezes AM. Fatores socioculturais e nível de atividade física no início da adolescência. Rev Panam Salud Pública 2007; 22:246-53.

44. Bastos JP, Araújo CL, Hallal PC. Prevalence of insufficient physical activity and associated factors in Brazilian adolescents. J Phys Act Health 2008; 5:777-94.

45. Tassitano RM, Bezerra J, Tenório MCM, Colares V, Barros MVG, Hallal PC. Atividade física em adolescentes brasileiros: uma revisão sistemática. Rev Bras Cineantropom Desempenho Hum 2007; 9:55-60.

46. Craig CL, Marshall AL, Sjostrom M, Bauman AE, Booth ML, Ainsworth BE, et al. International physical activity questionnaire: 12-country reliability and validity. Med Sci Sports Exerc 2003; 35:138195.

47. Armstrong T, Bull F. Development of the World Health Organization Global Physical Activity Questionnaire (GPAQ). J Public Health 2006; 14:66-70.

48. Troiano RP, Berrigan D, Dodd KW, Masse LC, Tilert T, McDowell M. Physical activity in the United States measured by accelerometer. Med Sci Sports Exerc 2008; 40:181-8.

49. Currie C, Roberts C, Morgan A, Smith R, Settertobulte W, Samdal O, et al. Young people's health in context. Health Behaviour in School-aged Children (HBSC) study: international report from the 2001/2002 survey. Geneva: World Health Organization; 2004. (Health Policy for Children and Adolescents, 4).
50. Haskell WL, Lee IM, Pate RR, Powell KE, Blair SN, Franklin BA, et al. Physical activity and public health: updated recommendation for adults from the American College of Sports Medicine and the American Heart Association. Circulation 2007; 116:1081-93.

51. Strong WB, Malina RM, Blimkie CJ, Daniels SR, Dishman RK, Gutin B, et al. Evidence-based physical activity for school-age youth. J Pediatr 2005; 146:732-7.

52. Gomes VB, Siqueira KS, Sichieri R. Atividade física em uma amostra probabilística da população do Município do Rio de Janeiro. Cad Saúde Pública 2001; 17:969-76.

53. Oehlschlaeger MHK, Pinheiro RT, Horta B, Gelatti C, San'tana P. Prevalência e fatores associados ao sedentarismo em adolescentes de área urbana. Rev Saúde Pública 2004; 38:157-63.

54. Dias-da-Costa JS, Hallal PC, Wells JCK, Daltoé T, Fuchs SC, Menezes AMB, et al. Epidemiology of leisure-time physical activity: a population-based study in southern Brazil. Cad Saúde Pública 2005; 21:275-82.

55. Pitanga FJG, Lessa I. Prevalência e fatores associados ao sedentarismo no lazer em adultos. Cad Saúde Pública 2005; 21:870-7.

56. Dumith SC, Gigante DP, Domingues MR. Stages of change for physical activity in adults from Southern Brazil: a population-based survey. Int J Behav Nutr Phys Act 2007; 4:25.

57. Barreto SM, Passos VM, Firmo JO, Guerra HL, Vidigal PG, Lima-Costa MF. Hypertension and clustering of cardiovascular risk factors in a community in Southeast Brazil: The Bambui Health and Ageing Study. Arq Bras Cardiol 2001; 77:576-81.

58. Lima-Costa MF, Barreto SM, Uchoa E, Firmo JO, Vidigal PG, Guerra HL. The Bambui Health and Aging Study (BHAS): prevalence of risk factors and use of preventive health care services. Rev Panam Salud Pública 2001; 9:219-27.

59. Gus I, Fischmann A, Medina C. Prevalence of risk factors for coronary artery disease in the Brazilian State of Rio Grande do Sul. Arq Bras Cardiol 2002; 78:478-90.

60. Lima-Costa MF. A escolaridade afeta, igualmente, comportamentos prejudiciais à saúde de idosos e adultos mais jovens? Inquérito de saúde da Região Metropolitana de Belo Horizonte, Minas Gerais, Brasil. Epidemiol Serv Saúde 2004; 13:201-8.

61. Doro AR, Gimeno SG, Hirai AT, Franco LJ, Ferreira SR. Análise da associação de atividade física a síndrome metabólica em estudo populacional de nipo-brasileiros. Arq Bras Endocrinol Metabol 2006; 50:1066-74.

Submitted on $10 / \mathrm{Feb} / 2009$

Final version resubmitted on 23/Jun/2009

Approved on 03/Jul/2009 